OnLine Journal of Biological Sciences 11 (2): 37-47, 2011

ISSN 1608-4217

(C) 2011 N. Bulyakova et al., This open access article is distributed under a Creative Commons Attribution

(CC-BY) 3.0 license

\title{
Laser Therapy of Irradiated Traumatized Skeletal Muscles and State of Immune System in Animals
}

\author{
${ }^{1}$ Nelly Bulyakova, ${ }^{2}$ Svetlana Zubkova and ${ }^{1}$ Valentina Azarova \\ ${ }^{1}$ Institute of Ecology and Evolution, Russian Academy of Sciences, \\ 119071, Leninskii pr. 33, Moscow \\ ${ }^{2}$ Russian Scientific Center of Restorative Medicine and Balneology, \\ MPH and SD RF, 121099, Borisoglebskiy, Str. 9, Moscow
}

\begin{abstract}
Problems statement: This study was designed to examine the effect of laser therapy on regeneration of traumatized skeletal muscles and on dynamics of immune responses in thymus and bone marrow under ionizing radiation. Approach: After local X-ray radiation of both rat gastrocnemius muscles at a dose $20 \mathrm{~Gy}$ or after total $\gamma$-irradiation of animals at a semiletal dose $6 \mathrm{~Gy}$, the muscles were damaged considerably as contractile organ (full cut across muscle). Then laser therapy was carried out with a helium-neon laser $(632.8 \mathrm{~nm})$ or infrared pulsed laser $(890 \mathrm{~nm}), 10$ treatments by 3 min during the first 15 or 30 days after trauma.The investigations were performed using histological, biochemical, cytological, cytogenetic and morphometric methods. Results: The received data showed that under ionizing radiation, He-Ne and IR laser irradiation stimulated the regeneration of skeletal muscles, improved the healing of skin-muscle wound and stimulated the prosesses of postradiation recovery in cells of the thymus and bone marrow. The regenerative activity of radiated traumatized muscles and the level of reduction of cytogenetic damages in bone marrow and the thymus varied depending on the period for which regenerative muscles were exposured to laser light, as well as the optical characteristics of lasers and the degree of radiation damages. The early appearance of reactive changes in structure of the thymus and the display them during 60 days point to increasing function of organ. Conclusion: Under local and total ionizing radiation and laser therapy of solely damaged skeletal muscles, positive dynamics of regeneration processes and improvement of cytological and cytogenetic indexes for main immune organs (the thymus and bone marrow) were observed. Herewith, the functional loading on thymus increased. We suggest that the findings should be considered in clinical practice.
\end{abstract}

Key words: Muscle tissue, posttraumatic regeneration, wound healing, thymus, bone marrow, ionizing radiation, laser therapy, radiation damages, antioxidant enzymes, membrane structures, cytogenetic disturbances, skeletal muscle

\section{INTRODUCTION}

Ionizing radiation suppresses the regenerative ability of skeletal muscle tissue. The proliferative activity of cells and intensity of biosynthetic and bioenergetic processes are significantly decreased. The revascularization and restoration of neuromuscular contacts in traumatized skeletal muscles are delayed. In area of skeletal muscle trauma, the skin-muscle ulcers appear. Under ionizing radiation, cytogenetic disturbances occur. The critical target cells are also membrane structures. The elevated lipid peroxidation leads to the disruption of the structure and function of biological membranes and the appearance of toxic products. Disbalance occurs in the redox processes.

The search for effective methods for stimulation of skeletal muscle recovery still remains an important problem in biology and medicine. There are experimental and clinical data that demonstrate the ability of low-intensity laser radiation to reduce the level of $x$-radiation damages and increase regenerative ability of organs and tissues. The positive effect of $\mathrm{He}-\mathrm{Ne}$ laser rays for the restoration of locally irradiated in doses 10-20 Gy damaged skeletal muscle as a contractile organ was shown in studies conducted by us and our colleagues formerly. Laser radiation

Corresponding Author: Nelly Bulyakova, Institute of Ecology and Evolution, Russian Academy of Sciences,

119071, Leninskii pr. 33, Moscow 
contributes to postradiation recovery in cells and tissues resulted in a decrease of aberrant mitoses (Stepanov et al., 1977). The laser radiation plays the role of structural antioxidant, preventing cellular structures from damage by reactive oxygen and stimulating the activity of antioxidant enzymes (catalase, superoxide dismutase, glutathione peroxidaze, ceruloplasmin) due to the resonant absorption of laser photons. Therapeutic effect of He$\mathrm{Ne}$ and IR lasers is the most widely studied.

However, there are discrepant data on the effect of the low-intencity laser radiation on the functional state of the main organs of immune system (the thymus and bone marrow). The immune system is known to control the processes of recovery of organs and tissues. The lymphoid cells realize not only immunologic but also morphogenetic control. In response to operative stress, the involutive changes in the thymus structure develop and the thymic peptides synthesize. They control the migration, proliferation, differentiation, apoptose of thymocytes and necessary to regulate the function of peripheral lymphoid organs. Recently it was shown that myoid cells of the thymus can migrate to the periphery and participate in the processes of posttraumatic regeneration of muscle tissue (Pagel et al., 2000). Pluripotent bone marrow stem cells may also participate in the regeneration of the tissues and in particular, differentiate into muscle cells, skin cells and release the angiogenic factors stimulating the formation of a network of blood vessels (Ferrari et al. 1998; Borue et al.,2004; McFarlin et al., 2006; Wu et al., 2007). The most of macrophages and fibroblasts in area of an inflammation have their origin from progenitor cells actively proliferating in the bone marrow (Michurina et al., 1991). Macrophages accomplish not only the function of phagocytosis, but have the ability to provide growth factors that stimulate the activity of myoblasts (Rappolee and Werb, 1992; Merly et al., 1999). Ionizing radiation reduces the immunological reactivity. The death of thymocytes, depletion and thymic atrophy result from progressive failure thymic epithelium (Yarilin, 1997). In the irradiated bone marrow, massive cell death, a high percentage of cells with chromosome aberrations, a disturbance of the process of cell renewal and exhaustion of adaptation reserves at the cellular level are observed.

According to literature data, the laser beams stimulate the proliferation and functional activity of lymphoid cells (Agaiby et al., 2000). Effect of He-Ne laser radiation increases the mitotic activity of bone marrow cells and stimulates hemopoiesis (Golovin et al.,
1978). However, there are data on decrease of activity of the immune system under the laser influence (Mester et al., 1978; Konchugova et al., 1992; Barilyak and Lopushan, 1981; Fedoseeva et al., 1988). The "breakdowns" in the structure of DNA of lymphoid cells, a decrease in secretory activity and suppression of natural killer cells were noted. The cases of increase of cytogenetic damages in bone marrow cells were observed.

In this study, we investigated the effect of radiation of $\mathrm{He}-\mathrm{Ne}$ and IR lasers on the posttraumatic regeneration of skeletal muscles subjected to ionizing radiation. Because the laser therapy for irradiated injured skeletal muscles is conducted on the background of immunosuppressive effect caused by ionizing radiation, the investigations of the state of the thymus and bone marrow under these circumstances were also carried out. Exposure to ionizing radiation has been a local or general. Besides, the dynamics of healing of skin-muscle wound was recorded.

\section{MATERIALS AND METHODS}

The experiment was performed on the outbred adult male rats (aged 2-3 months). Rats were subjected to local or total effect of ionizing radiation. Then the animals were anesthetized with nembutal and under aseptic conditions, the skin and covering muscle on both hind legs were dissected and the gastrocnemius muscles were transverse cut in the middle. The neurovascular bundle, which lies on the surface of gastrocnemius muscle and peroneal nerve lying under muscle were kept intact. Then the covering muscle and skin at the each leg were sewed with surgical silk.

After surgery, the each hind limb of rat was exposed to laser rays in the projection area of gastrocnemius muscle. In controls, irradiated traumatized muscles regenerated without laser therapy.

Conditions for a local X-ray irradiation: an RUP200 device (Russia); voltage, $190 \mathrm{kV}$; current, $15 \mathrm{~mA}$; dose power, $0.75 \mathrm{~Gy} / \mathrm{min}$; filters, $0.5 \mathrm{~mm} \mathrm{Cu}, 1 \mathrm{~mm} \mathrm{Al}$; a dose of $20 \mathrm{~Gy}$. Conditions for a total $\gamma$ - radiation: a GUBE device (Russia); the source of 60Co irradiation; dose power, $0.6 \mathrm{~Gy} / \mathrm{min}$; time of radiation, $10 \mathrm{~min}$; a dose of $6 \mathrm{~Gy}$ (semiletal dose for rats). Conditions for He-Ne laser exposure: an OKG-12 device (Russia); the wavelength, $0.63 \mu \mathrm{m}$; the laser ray was defocused using a lens; diameter of radiation field was $2-2.5 \mathrm{~cm}$; power density on the irradiated surface was equal to 2.5-3.0 $\mathrm{mW} \mathrm{cm}^{-2}$. Conditions for impuls IR laser exposure: 
"Orion" device (firm "Zhiva", Russia); the wavelength, $0.89 \mu \mathrm{m}$, the carrier frequency, $1500 \mathrm{~Hz}$; length of pulse, $7 \times 10^{-8} \mathrm{c}$; pulse power, $4.8 \mathrm{~W}$; modulation frequency, $10 \mathrm{~Hz}$; the duration of modulating pulse, $2 \times 10^{-2} \mathrm{c}$; the average pulse power, $0.002 \mathrm{~W}$; area of exposure, $1.5 \mathrm{~cm}^{2}$; the contact method.

Investigated organs and tissues were analyzed within 60 days after surgery using the histological, biochemical, morphometric, cytological and cytogenetic methods. Muscles and the thymus were treated according to the universally accepted histological methods: fixed in Carnoy's mixture, paraffin sections of thickness 7-8 $\mu \mathrm{m}$ were stained with hematoxylin according to Regau's method and additionally stained according to Mallori's method. Pieces of bone marrow tissue were removed from the tibia, stained with acetocarmine and the squash preparations were prepared in a drop of chloral hydrate.

When tested the muscle regenerates, the morphometric method was used to estimate the amount of muscle and connective tissues per longitudinal histology section (by measuring the areas occupied by these tissues on a section in \%). Then, data obtained in the experiment, expressed as a percentage of the relevant indicators in the control group. The extent of destruction in muscle tissue and the inflammation were assessed by the activity of lysosomal enzyme elastase and $\alpha 1$-proteinase inhibitor ( $\alpha 1-\mathrm{PI})$. Destructive processes at the membrane level assessed by lipid peroxidation (LPO) and total antioxidant activity (AOA). State of microcirculatory processes in the muscle was determined by the activity of $\alpha 2$ macroglobulin ( $\alpha 2-\mathrm{MG})$. In addition, the activity of elastase, $\alpha 1-\mathrm{PI}$ and $\alpha 2-\mathrm{MG}$ were detected in the blood serum, which allowed to judge about the laser influence of not only on the regenerative processes in the muscle, but also on the level of microcirculation and destructive processes in the body as a whole. When studied the functional activity of the thymus, the amount of cortical and medullar substances in the thymus was measured by the "method of fields" using a test ocular grid and an MBS-1 binocular lens (LOMO, Russia), x25 magnification. Counting was carried out only in the lobules, retaining its structure. The number of points in the cortical and medullar layers was expressed as a percentage of the total number of points in the entire histological section of the thymus. Besides, we estimated the specific activity of the thymocyte genome determined as the product of the number of thymocyte nuclei (calculated per unit of thymus weight) by the intensity of incorporation of the dye Acridine Orange (AO) into the deoxyribonucleoproteid (DNP) of each nucleus. Dye incorporation was measured photometrically at $492 \mathrm{~nm}$ with a "Perkin Elmer" spectrophotometer. Thymocyte nuclei (TM) were counted with a "Picoscale" photoelectron particle counter.

For cytological monitoring of cells of the thymus, the mitotic index of the thymocytes (MI, \%o) and the number of lymphocytes with karyopycnosis (\%) were determined. The total number of cells calculated in 6-8 histological sections was equal to 3000-4000. Cytogenetic monitoring of cells of the thymus and bone marrow were carried out by the anaphase method. It was tested 100 late anaphases and early telophases. When determining the percentage of cell with chromosome aberrations, the mitoses with bridges, fragments, the mixed pathology (bridges and fragments) and others aberrations (with sticky chromosomes and significantly lagging) were into account. We also determined the relative mass of the thymus (the ratio between the thymus and body mass expressed in per cent). The thymus and bone marrow of intact rats were studied.

Three-six animals were used at each period of study. The animals were taken out of the experiment with injection of large doses of nembutal. Quantitative data were processed statistically and differences between mean values were estimated using Student's $t$ test.

\section{RESULTS}

Effect of He-Ne laser on the skeletal muscles after local radiation in a dose of $20 \mathrm{~Gy}$ and trauma: The regimen of laser exposure: 10 treatments for $2 \mathrm{~min}$ each during the first 15 days (daily except on weekends) or during 30 days (for 2-3 treatments per week) after Xirradiation and injury. Power density on the irradiated surface of shin was $2.5-3.0 \mathrm{~mW} \mathrm{~cm} \mathrm{~cm}^{-2}$. The total dose per course of laser therapy one limb was equal to 3.0$3.6 \mathrm{~J} \mathrm{~cm}^{-2}$, in general, for each animal $6.0-7.2 \mathrm{~J} \mathrm{~cm}^{-2}$.

Muscle: Under the influence of He-Ne laser on muscle for the first 15 days after injury, signs of postradiation recovery in tissues were revealed. The connective tissue consisted of more quantity of cellular elements and collagen fibers. The regeneration of muscle tissue was observed in septum from the ends of unsected muscle fibers and on the edges of both muscle stumps from the ends of transected muscle fibers. Muscle fibers regenerating from the proximal and distal stumps grew into the area of injury. Bundles of collagen fibers arranged longitudinally. As shown by morphometric and biochemical data (Fig. 1), the destructive processes in muscle tissue of the regenerates were less pronounced. 
OnLine J. Biol. Sci., 11 (2): 37-47, 2011

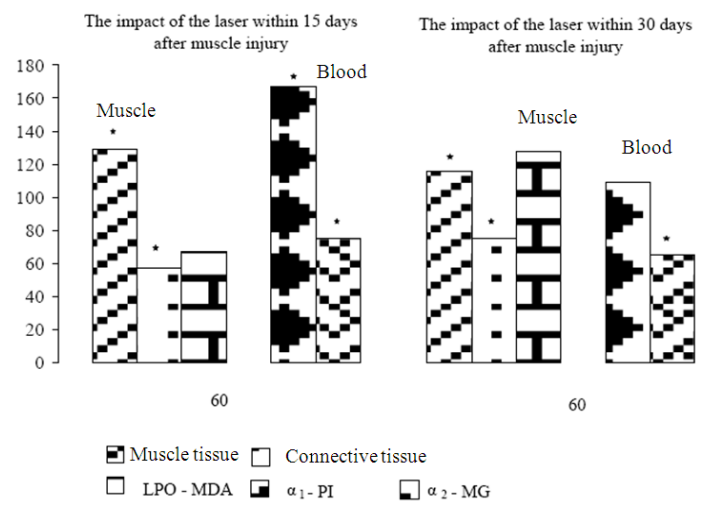

Fig. 1: Morphometric and metabolic indices of irradiated $(20 \mathrm{~Gy})$ muscle regenerates and blood serum under the influence of $\mathrm{He}-\mathrm{Ne}$ laser at a dose of 3.0 - $3.6 \mathrm{~J} \mathrm{~cm}^{-2}$ for each operated rat leg. The term of recovery was 60 days after injury of the muscles. Note: Y-axis - percentage of the average values of indices of the relevant parameters in the control (regeneration of irradiated muscles without laser exposure). *: Significant differences compared with controls

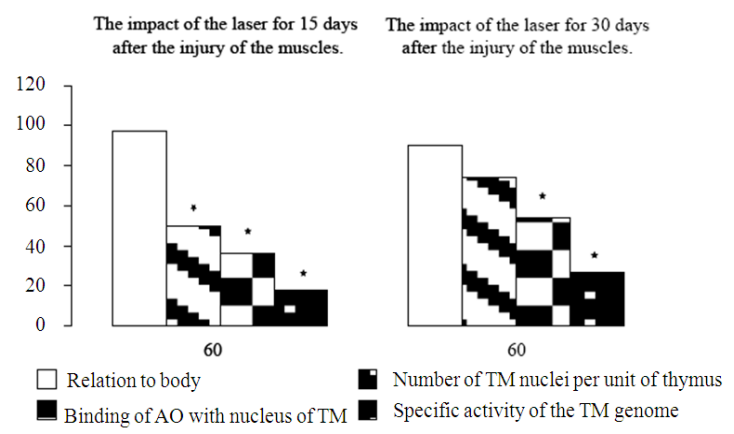

Fig. 2: Indicators of the functional state of the thymus under the influence of He-Ne laser at a dose of 3.0$3.6 \mathrm{~J} \mathrm{~cm}^{-2}$ for each irradiated (20 Gy) and operated rat leg. The term of recovery was 60 days after the injury of the muscles. Note: Y-axis-percentage of the average values of indices of the relevant parameters in the control (regeneration of irradiated muscles without laser exposure). *: Significant differences compared with controls

The quantity of muscle tissue in them was $129 \%$ of control $(59.7 \pm 1.7 \%$ in control and $77.0 \pm 4.5 \%$ in the experiment, $\mathrm{p}<0.01)$. Reduction the level of the lipid peroxidation to $67 \%$ from the corresponding control, as well as increase activity of $\alpha 1-$ PI to $167 \%(p<0.02)$ in blood serum indicated on the positive dynamics of the recovery process. The activity of $\alpha 2-\mathrm{MG}$ decreased to $75 \%(\mathrm{p}<0.05)$, that is an indication of the increase in activity of blood coagulation and the decrease in permeability of blood vessels, as well as some increase in activity of the immune system.

The prolonged fractional radiation of He-Ne laser at the same dose was less effective. Under influence of $\mathrm{He}-\mathrm{Ne}$ laser on muscle within a month, the regeneration of muscle fibers was also observed but muscle fibers did not sprout deeply into the wound. The space between the two muscle stumps was filled with connective tissue containing bundles of narrow collagen fibers and areas of loose connective tissue with a small amount of leukocytes, macrophages, mast cells and fragments of myofiber debris. The quantity of muscle tissue in the irradiated traumatized muscle was equal to $117 \%$ of control $(59.7 \pm 1.7 \%$ in control and $69.6 \pm 0.9 \%$ in the experiment, $\mathrm{p}<0.01)$. The trend towards increasing LPO in tissues of regenerate by $28 \%$ compared to the control point to the fact that the balance between the oxidation of membrane structures in cells and the activity of endogenous antioxidants have not yet fully recovered. In the blood serum activity of $\alpha 1$-PI was not reliably different from control values. Decrease in activity of $\alpha 2-$ MG by $35 \%(p<0.01)$ suggests that the state of the vascular system in regenerating tissues and activity of the immune system were much the same when exposed to rays of $\mathrm{He}-\mathrm{Ne}$ laser during two weeks.

Thymus: Under the effect of He-Ne laser for 15 days after the injury of muscles, a decrease in functional activity of the thymus was noted (Fig. 2). On 60th day of research, relative mass of the thymus did not differ from control. The number of nuclei of thymocytes was $50 \%$ of control, the binding of dye $\mathrm{AO}$ in thymocyte nuclei and the specific activity of the genome of thymocytes decreased as compared with the control to 36 and $18 \%$ respectively ( $\mathrm{p}<0.01$ ).

Under the influence of He-Ne laser irradiation on the injured muscles during a month, there was a tendency to decrease the relative mass of the thymus and the number of nuclei of thymocytes. Decrease in the specific activity of thymocytes and the ability of their nuclei to bind AO dye were significant $(p<0.01)$.

Bone marrow: Effect of He-Ne laser irradiation in both regimens promoted the processes of postradiation recovery in bone marrow cells that resulted in reducing the percentage of mitoses with chromosome aberrations (Fig. 3). In the controls, the number of cells with chromosomal aberrations was equal to $52.3 \pm 0.6 \%$, with laser therapy for 15 days $-44.5 \pm 1.2 \%(\mathrm{p}<0.01)$, while under the influence of the laser for 30 days $-45.8 \pm 1.0 \%$ $(\mathrm{p}<0.01)$. Qualitative composition of chromosomal aberrations did not suffer significant changes. 
OnLine J. Biol. Sci., 11 (2): 37-47, 2011

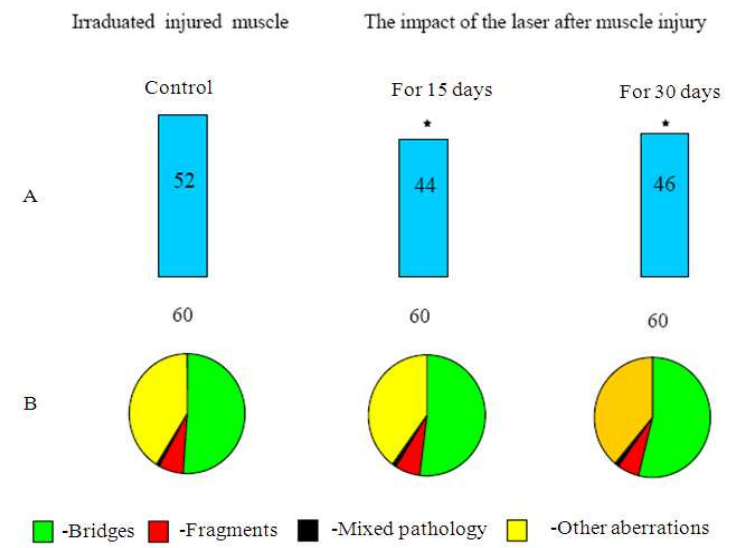

Fig. 3: Percentage of aberrant mitoses (A) and their qualitative composition (B) in rat bone marrow cells under the influence of $\mathrm{He}-\mathrm{Ne}$ laser at a dose of 3.0-3.6 J/ $\mathrm{cm}^{2}$ for each irradiated (20 Gy) and operated leg. The term of recovery was 60 days after the injury of the muscles. *: Significant differences compared with controls

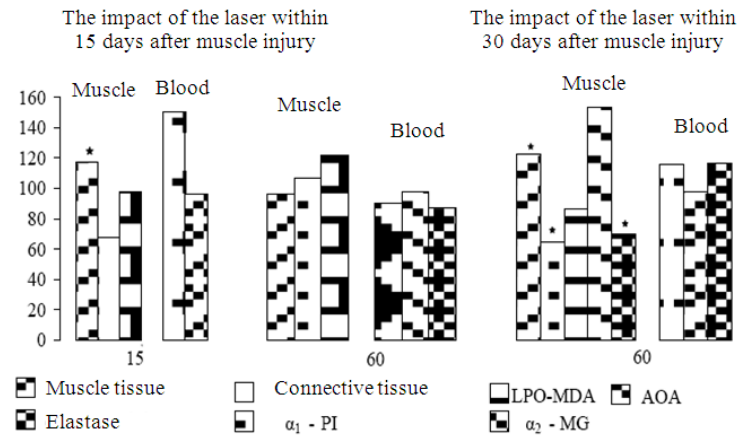

Fig. 4: Morphometric and metabolic indices of irradiated (20 Gy) muscle regenerates and blood serum under the influence of IR laser at a dose of $3.6 \mathrm{~J} / \mathrm{cm}^{2}$ for each operated rat leg. The terms of recovery were 15 and 60 days after the injury of the muscles. Note: Y-axis-percentage of the average values of indices of the relevant parameters in the control (regeneration of irradiated muscles without laser exposure). *: Significant differences compared with controls

Skin: Laser therapy of irradiated injured gastrocnemius muscles for 15 days was more effective for the healing of skin-muscle wounds. By the 60 th day, in most cases, skin sutures were clean and dry on the operated legs. Only in $13 \%$ cases, the skin sutures were not completely healing on surgery shins, but suppuration was not observed. In the conditions of laser therapy for 30 days, skin-muscle ulcers also did not appear by the end of the study (60 days), but $60 \%$ of the skin sutures was not completely grew together.

Effect of IR laser on the skeletal muscles after local radiation in a dose of $20 \mathrm{~Gy}$ and trauma: Two regimens of laser action were also investigated. The dose of pulsed infrared laser radiation was almost the same as with a He-Ne laser.

The total dose per course of laser therapy for one limb was $3.6 \mathrm{~J} \mathrm{~cm}^{-2}$, in general, for each animal $7.2 \mathrm{~J} \mathrm{~cm}^{-2}$.

Muscle: When exposed to infrared laser radiation within the first 15 days after injury, both muscle stumps grew together poorly.

In the field of trauma of 15-day regenerates, infiltration of lymphocytes, monocytes and macrophages were observed. The quantity of fibrin was equal to $71 \%$ of control $(p<0.01)$. Some signs of recovering in the granulation tissue may be noted. There were a greater number of spindle-shaped fibroblasts, mitosis and collagen fibers. Network of blood vessels actively formed. The accumulations of large oval nuclei at the ends of transected muscle fibers were visible. Many of them had large nucleoli. Greater number of growing muscle tubules was observed in the septum. However, fibrin and tissue degradation products in area of muscle trauma impeded their growth. In the 60-day regenerates, the dense connective tissue formed. There was a more extensive network of capillaries. Hemorrhages were observed. The regions of regenerating muscle tissue were represented by narrow muscle fibers with macrophages and mast cells between them. There were muscle fibers exposed to Zenker's necrosis. Regenerating muscle tissue did not fill the damaged area in the muscle. Under daily exposure to infrared laser light in the early stages of regeneration of irradiated muscles (Fig. 4), after complication of laser therapy (by the 15th day), the quantity of muscle tissue increased up to $117 \%(64.0 \pm 3.2 \%$ in control and $74.9 \pm 0.9 \%$ in the experiment, $\mathrm{p}<0.02)$. A reaction of inflammation accelerated. The level of LPO in regenerating tissues did not exceed that of control, but increase of $\alpha 1$-PI in blood serum (by 50\%) indicated on anti-inflammatory action of laser radiation. By the end of the observed period (60 days, i.e., through 1.5 months after completion of laser therapy) the quantity of muscle tissue in the regenerates did not differ from control. The tendency to a rise in LPO was noted. The activity of $\alpha 1-\mathrm{PI}, \alpha 2-\mathrm{MG}$ and elastase in the blood serum remained at a level of control. 


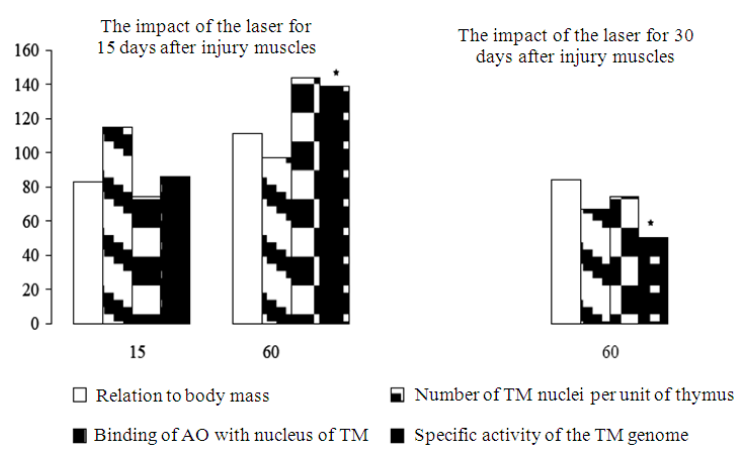

Fig. 5: Indicators of the functional state of the thymus under the influence of infrared laser exposures at a dose of $3.6 \mathrm{~J} \mathrm{~cm}^{-2}$ for each irradiation (20 Gy) and operated rat limb. Terms of recovery were 15 and 60 days after the injury of the muscles. Note: Y-axis-percentage of the average values of indices of the relevant parameters in the control (regeneration of irradiated muscles without laser exposure). *: Significant differences compared with controls

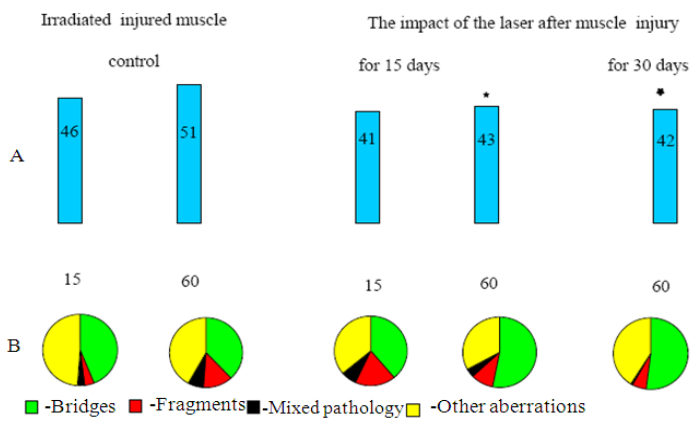

Fig. 6: Percentage of aberrant mitoses (A) and their qualitative composition (B) in rat bone marrow cells when exposed to infrared laser at a dose of $3.6 \mathrm{~J} \mathrm{~cm}^{-2}$ for each irradiated $(20 \mathrm{~Gy})$ and operated leg. Terms of recovery were 15 and 60 days after the injury of the muscles. *: Significant differences compared with controls

Under conditions of prolonged IR laser radiation (during the first month after muscle injury), both muscle halves grew together stronger. Regenerating muscle tissue was observed at the edge of the proximal and distal stumps, in the septum and deeply in area of injury. At the edge of muscle stumps, regenerating fibers located densely. The area occupied by loose connective tissue, cells of the lymphoid tissue and macrophages was reduced.
The dilatation of large blood vessels and extensive network of capillaries were noted. In the 60-day regenerates, about of $123 \%$ of muscle tissue was preserved $(59.7 \pm 1.7 \%$ in the control and $73.7 \pm 3.6 \%$ in the experiment, $\mathrm{p}<0.05)$. The destructive changes were declined in regenerating muscles. The increase antioxidative activity (AOA) by $54 \%$ and reduction of Lipid Peroxidation (LPO) by $13 \%$ compared with controls pointed to stability of membrane systems in muscle. The activity of lysosomal proteolytic enzyme elastase in tissues of regenerates decreased by $30 \%$ $(9.2 \pm 0.8 \%$ in control and $6.4 \pm 0.5 \%$ in the experiment, $\mathrm{p}<0.02$ ) and had tendency to increase in blood serum, but it seems to be compensated by some increased activity of its inhibitor ( $\alpha 1-\mathrm{PI})$. Activity of $\alpha 2-\mathrm{MG}$ did not differ from control.

Thymus: Under IR laser exposures for the first two weeks after muscle trauma, no changes were detected in any of the studied parameters (Fig. 5). One can only note a tendency for decline in mass of the thymus, the capacity of the nuclei in thymocytes to binding with AO dye and specific activity of thymocyte genome, whereas the number of nuclei of TM increased slightly $(15 \%)$. After 60 days, the mass of the thymus and the number of nuclei in thymocytes approached to control. However, the activity of thymocytes remained: a tendency to increase in ability of thymocyte nuclei to bind the AO dye (144\%) and in specific activity of the TM genome $(139 \%, \mathrm{p}<0.1)$ compared with controls.

Under IR laser exposures for a month, after 60 days, the functional activity of the thymus remained significantly reduced. So, the thymus mass, a number of nuclei of thymocytes and their capacity to bind the AO dye were 84,67 and $74 \%$ of controls, respectively and the specific activity of the TM genome decreased by $50 \%(101.4 \pm 15.9$ rel. units in the control and $50.4 \pm 7.4$ rel. units in the experiment, $\mathrm{p}<0.02$ ). Decrease in functional activity of the thymus under the influence of infrared laser radiation for 30 days was more pronounced than that of under exposures for 15 days.

Bone marrow: When IR laser therapy was carried out in the early stages of regeneration, after its completion a tendency to reduce the number of mitoses with chromosomal aberrations to $41.0 \pm 1.6 \%$ compared with control was observed. But in this case, the elevated number of mitoses with fragments (18\%) and "mixed pathology" (7\%) pointed to more strong cytogenetic damages in bone marrow cells (Fig. 6). 
After 60 days, the percentage of aberrant mitoses increased non-significantly compared with the 15-day period, but it was reliably lower than in the corresponding period in the control $(43.2 \pm 2.7 \%, \mathrm{p}<0.05)$. A number of mitoses with fragments and "mixed pathology" were reduced to 10 and $4 \%$, respectively. The most of aberrant mitoses was consisted of the mitoses with bridges and "other" aberrations.

When laser therapy was carried out for month, after 60 days, the reduced quantity of chromosomal aberrations, as well as mitoses with fragments and "mixed pathology" were also observed. It should be noted that the aberrant mitoses with the formation of several bridges (2-4) and centrioles were revealed in both regimens of infrared laser radiation.

Skin: The positive effect of IR laser therapy on healing of skin-muscle wounds was dependent on laser radiation regimen. When laser therapy carried out during the first 15 days, after the sessions, the ulcers, erythema and maceration of the skin were observed only in $30 \%$ of cases. Further, the full healing of skinmuscle wounds did not occur. No signs of fester were noted, but $50 \%$ of the wounds were covered with a thin layer of the epithelium, or the edges of skin wounds grew together incompletely. Laser therapy of the damaged muscles during the month was more effective. By the end of the observed period (60 days), the formation of radiation ulcers in the wounds did not note at all and incomplete healing of skin sutures was observed only in $8.3 \%$ of cases, therewith without septic complications. Skin sutures were dry and clean. The growth of hairs on operated legs was improved.

Effect of He-Ne laser on the traumatized skeletal muscles of rats after total body radiation at a dose of 6 Gy: Two regimens of $\mathrm{He}-\mathrm{Ne}$ laser exposures were also studied but a dose of laser irradiation was elevated. The course consisted of 10 treatments by $3 \mathrm{~min}$, a total dose of $4.5-5.4 \mathrm{~J} / \mathrm{cm}^{2}$ on the each operated hind leg and $9.0-10.8 \mathrm{~J} / \mathrm{cm}^{2}$ per animal as a whole.

Muscle: Under the action of laser beams during the early stages of regeneration, the proximal and distal stumps in 15-day regenerates grew together along the perimeter of the cross-section. A reaction of inflammation and resorption of the fibrin and products of tissue degradation occurred actively. The number of multinuclear macrophages decreased significantly. The connective tissue was restored as indicated by active proliferation of fibroblasts, endothelial cells and formation of collagen fibers. The areas of loose connective tissue with lymphocytes and mast cells were reduced. The laser radiation stimulated regenerative ability of muscle tissue. Muscle fibers grew into the area of injury. The amount of muscle tissue was equal to $116 \%(72.2 \pm 2.2 \%$ in the control and $83.8 \pm 1.9 \%$ in the experiment, $\mathrm{p}<0.01)$ at 15 days and to $110 \%(76.2 \pm 2.4 \%$ in controls and $84.2 \pm 1.1 \%$ in the experiment, $\mathrm{p}<0.01$ ) at 30 days (Fig. 7). The differentiated muscle fibers and myotubes occupied the area of damage. Leukocyte infiltration, macrophages and mast cells were observed among the regenerating muscle fibers. Further, the quantity of muscle tissue decreased.

In conditions of prolonged exposure to laser radiation, both muscle stumps were firmly grew together. The resorbtion of the products of tissue decomposition was accelerated, region of trauma was contracted. The regenerative ability of connective tissue was significantly restored. An active growth of muscle tissue from the ends of transected muscle fibers and in the septum, from the ends of non-transected muscle fibers occured. The mitotically dividing cells were frequently observed in the vicinity of the muscle fibers. A muscular type of structure of the regenerates was remained throughout the study period. The amount of muscle tissue in them after 15,30 and 60 days was significantly higher than in the controls $(p<0.02, p$ $<0.01, \mathrm{p}<0.02$, respectively).

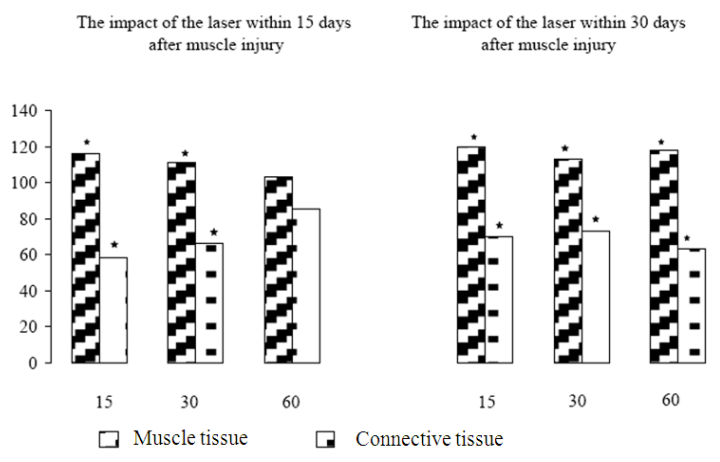

Fig. 7: Morphometric parameters of muscle regenerates in irradiated $(6 \mathrm{~Gy})$ rats under the influence of $\mathrm{He}-\mathrm{Ne}$ laser at a dose of $4.5-5.4 \mathrm{~J} / \mathrm{cm}^{2}$ for each operated leg. Terms of regeneration were 15,30 and 60 days after muscle injury. Note: Y-axis percentage of the average values of indices of the relevant parameters in the control (regeneration of irradiated muscles without laser exposure). *: Significant differences compared with controls 


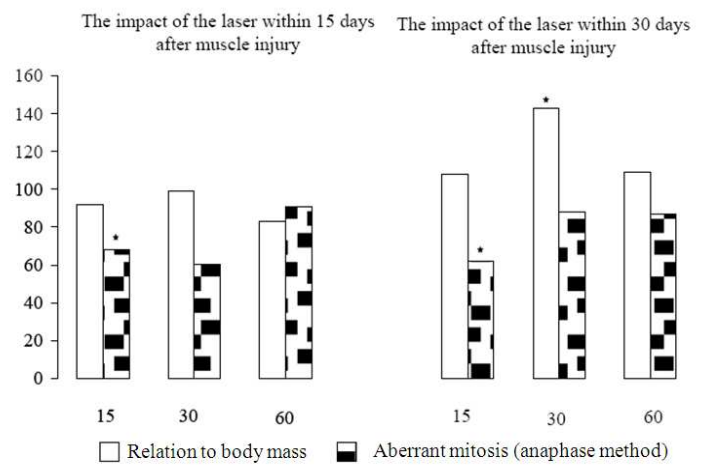

Fig. 8: Indicators of the functional state of thymus in the irradiated $(6 \mathrm{~Gy})$ rats under the influence of $\mathrm{He}-\mathrm{Ne}$ laser at a dose of $4.5-5.4 \mathrm{~J} / \mathrm{cm}^{2}$ for each operated leg. Terms of regeneration were 15,30 and 60 days after the injury of the muscles. Note: Y-axis - percentage of the average values of indices of the relevant parameters in the control (muscle regeneration in irradiated rats without laser exposure). *: Significant differences compared with controls. In the control after 30 and 60 days, the thymus was studied in 2 rats for each term. The rest rats died

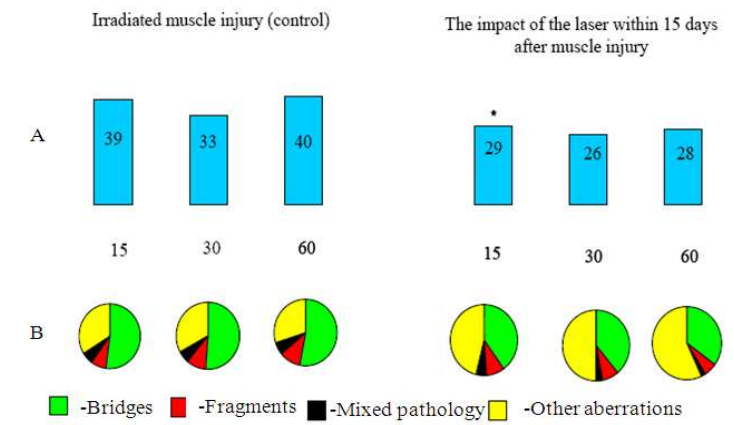

Fig. 9: Percentage of aberrant mitoses (A) and their qualitative composition (B) in bone marrow cells in rats irradiated at a dose of $6 \mathrm{~Gy}$ when exposed to $\mathrm{He}-\mathrm{Ne}$ laser at a dose of $4.5-5.4 \mathrm{~J} / \mathrm{cm}^{2}$ for each operated leg. Terms of regeneration were 15,30 and 60 days after injury of the muscles. *: Significant differences compared with controls

Thymus: When the laser therapy of muscles at intensive regimen (every day for the first 15 days after surgery), the acceleration of aplasia of the thymus, enlarge of medullar layer in the lobules, the involution of the layers, an increase of agglomeration of thymocytes, lysis and pycnosis of the nuclei of lymphoid cells in the thymus clearly defined to time of the completion of laser exposures. And in each animal, these changes have occurred with varying intensity. Then the histological structure of the thymus is gradually recovering and to the 60th day after surgery, did not differ from that of the thymus in control. The thymus mass decreased and remained at a lower level than in the control (no laser) during the 60 days (Fig. 8). Laser radiation promoted to decrease of mitoses with chromosomal aberrations. By to time of completion of treatment, the number of aberrant mitoses was equal to $70 \%(41.0 \pm 2.1 \%$ in control and $28.0 \pm 4.0 \%$ in the experiment, $\mathrm{p}<0.05)$. Further, the number of aberrant mitoses was elevated and approached control.

In conditions of prolonged laser irradiation, reactive changes in the thymus occurred slowly and gradually intensified to the 30th day (by the time of completion of laser therapy). After 60 days, the structure disturbances took place in many of lobes but lymphoid cells were compactly located in the cortical layer. The mitoses and lymphocytes with pyknotic nuclei were frequently found. The positive effect of prolonged therapy with $\mathrm{He}-\mathrm{Ne}$ laser was more visible. So, already after the five procedures (to 15th day), a quantity of aberrations was equal to $62 \%$ of control $(16 \pm 0.9 \%$ in experiment and $26 \pm 1.5 \%$ in control, $\mathrm{p}<0.01)$. By the end of the observation, the quantity of chromosomal aberrations progressively increased but still remained at 12 and $13 \%$ lower compared with control.

Bone marrow: The effect of He-Ne laser radiation on bone marrow cells was studied only in terms of laser therapy of injured muscles for the initial stages of regeneration (within the first 15 days after the injury of the muscles). The data received showed that the processes of postradiation recovery in bone marrow cells were stimulated (Fig. 9). After completion the laser therapy, the number of cells with chromosomal aberrations decreased from $39.1 \pm 0.8 \%$ in the control to $28.8 \pm 1.5 \%$ in the experiment, $\mathrm{p}<0.01$. Further, lower level of chromosomal aberrations in irradiated rats was maintained. Herewith, by the end of the observed period (60 days), the number of aberrant mitoses with fragments and "mixed pathology" (bridges with fragments) reduced in 2-3 times.

Skin: Rays of He-Ne laser improved the healing of skin-muscle wound. To the period of completion of intensive laser therapy (every day for 15 days after surgery) the deteriorations of healing of skin-muscle wounds were observed in 4 of 18 operated legs. However, these were only small superficial abscesses in 
the skin suture. Musculocutaneous ulcer was appeared only in one case, but the muscle regenerate was preserved. By the end of the observed period, the deteriorations in the wound healing were not observed. Sutures were clean and smooth. When the more prolonged He-Ne laser therapy of both rat hind limbs, any complications in the healing of skin-muscle wounds were not observed. To 60 days after surgery, the small scabs on the skin were observed alone on two operated legs from the 18.

In addition to the above described observations, we noted that effect of $\mathrm{He}-\mathrm{Ne}$ laser on operated legs during the first 15 and 30 days after trauma muscles increased survival of rats radiated at a dose of $6 \mathrm{~Gy}$. In control series (without laser treatment), about $50 \%$ of the animals died.

\section{DISCUSSION}

The received data showed that the emission of helium-neon and infrared pulsed lasers in studied doses and regimens has a positive effect on the regeneration of skeletal muscles exposured to ionizing radiation. The increase of proliferative activity in muscle elements, the acceleration of inflammation and resorption of production of tissue destruction, the reduction in the level of lipid peroxidation in membrane structures of cells (LPO) and a relatively high level of Antioxidant Activity (AOA), decrease of activity of lysosomal proteolytic enzyme elastase, as well as high activity of $\alpha 1-\mathrm{PI}$ and $\alpha 2-\mathrm{MG}$ were observed in tissues of regenerates and in blood serum. The muscle tissue was predominanted in structure of regenerates. Laser therapy of injured skeletal muscles improved the healing of skin and underlying soft tissues in the irradiated operated legs of the animal. Our observations are consistent with the literature data received on non-exposured to ionizing radiation injured skeletal muscles. It was shown that the determining factor in the stimulation of regenerative capacity of muscle tissue under the action of laser beams is the increase the activity of "dormant muscle satellite cells" and accelerated entry of cells into the reproductive cycle (Shefer et al., 2001; Shefer et al., 2002; Doin-Silva et al., 2009). The decrease of destructive processes in the injured muscle tissue and apoptosis of muscle cells, increase of the muscle fibers viability after laser radiation resulted from the enhance activity of $\mathrm{Bcl}-2$ protein and the reduction of the protein $(\mathrm{BAX})$ that causes apoptosis.

At the same time, our observations showed that the efficiency of He-Ne and IR laser action in studied doses and regimens depended on the period during which the irradiated regenerating muscle subjected to laser exposures. According to morphometric and biochemical data, the therapy with $\mathrm{He}-\mathrm{Ne}$ laser at a dose of 3.0-3.6 $\mathrm{J} / \mathrm{cm}^{2}$ per each hind leg was more preferred during the first 15 days after muscle injury (the most increase in muscle tissue by $29 \%$ ). The therapy with IR laser at the same dose was more effective by 2-3 treatments per week during the first 30 days after muscle trauma (the most increase in muscle tissue by $17 \%$ ). Under a total gamma-irradiation of animals and $\mathrm{He}-\mathrm{Ne}$ laser radiation at a dose 4.5-5.4 J/ $/ \mathrm{cm}^{2}$, the prolonged (30 days) fractional regimen of exposures was also more effective. In 60-day regenerates, the amount of muscle tissue increased by $3.3 \%$ when laser therapy was carried out for two weeks and by $18 \%$ when that was performed during the month as compared to the controls.

Revealed features of recovery of irradiated muscle regenerates under influence $\mathrm{He}-\mathrm{Ne}$ and IR lasers apparently depended not only upon the optical properties of laser radiation but the degree of ionizing radiation damage to tissues and the organism as a whole. Laser therapy is effective when it was carried out at functionally reversible stages of pathological disorders, with partial preservation of a pool of progenitor muscle cells, satellite cells and myoblasts, which are cambial reserve for muscle tissue regeneration. In our early studies, we have demonstrated the absence of stimulating effect of He-Ne laser beams in adult rats after an increase of the local Xradiation dose up to 30-40 Gy. In old rats, a similar effect was observed at a dose of $20 \mathrm{~Gy}$. To obtain satisfactory results, a combination of laser therapy with tissue one was been required. The tissue therapy represents an autoimplantation of minced muscle tissue into area of muscle trauma. This provides an added source of muscle satellite cells, which are capable to regeneration.

Laser therapy of radiated damaged skeletal muscles in studied doses and regimens exerted the positive effect on postradiation recovery in cells of bone marrow and the thymus. When radiated of traumatized skeletal muscles locally at a dose of $20 \mathrm{~Gy}, \mathrm{He}-\mathrm{Ne}$ and IR laser radiation in total dose of $6.0-7.2 \mathrm{~J} / \mathrm{cm}^{2}$ for a rat reduced a number of mitoses with chromosomal abnormalities in cells of bone marrow. Effect was observed at both regimens and during all period of investigation (60 days). The stimulating effect of infrared laser rays on the processes of postradiation recovery in bone marrow cells was higher than that of He-Ne laser rays, possibly as a result of deeper penetration into the tissues. In rats irradiated with a dose of $6 \mathrm{~Gy}$, laser therapy of injured skeletal muscle in total dose of $9.0-10.8 \mathrm{~J} / \mathrm{cm}^{2}$ for the 
rat also had a positive impact. Immediately after completion of laser therapy a number of bone marrow cells with chromosomal aberrations was significantly decreased and remained at that level for a prolonged period. The activation of postradiation recovery in bone marrow cells may contribute to reduced formation of the radiation ulcers in the operated legs. Bone marrow stem cells, as established, are able to differentiate into skin cells and to form a network of blood vessels, thus taking part in the healing of skin-muscle wounds (Borue et al., 2004; McFarlin et al., 2006; Wu et al., 2007; Michurina et al., 1991).

The reaction of the thymus in response to operation stress and laser irradiation was more pronounced and occurred over a long period as compared with controls. After exposure of irradiated at dose 20 Gy muscles to radiation of $\mathrm{He}-\mathrm{Ne}$ and infrared lasers, all studied indexes of thymus functional activity were reduced by 60 days both at the laser exposure for two weeks and within a month. Under effect of $\mathrm{He}-\mathrm{Ne}$ laser rays and total irradiation of rats at a dose of $6 \mathrm{~Gy}$, reactive changes in the thymus differed depending on the period of laser therapy. Under two week laser treatment, the percentage of cells with chromosomal aberrations decreased significantly compared with controls and only on the 60 th day, the number of pathological mitoses gradually increased and approached the control value. In conditions of prolonged laser exposure, the changes in the thymus were expressed to a much less extent (more smooth reduction of thymus weight, more slow development of aplasia, as well as less intensive changes in its histological structure) as compared with laser exposures in the same dose during the first 15 days after muscle injury. But the processes of postradiation recovery in thymocytes were less stable. The number of thymocytes with chromosomal abnormalities increased gradually already during treatment (30 days) and on the 60th day also approached control.

The decrease of cytogenetic damages in cells of the thymus and bone marrow resulted from the stimulative effect of laser radiation on the immune system organs (Bulyakova et al., 2002). The early appearance of reactive changes in structure of the thymus and the display them for an extended period of muscle regeneration point to increasing function of organ. Based on these data, it was suggested that the positive dynamics of development of the regeneration process in injured muscles (fast purification of muscle wound, recovery of connective tissue, increase cell proliferation activity and stimulation of the regenerative ability of muscle tissue) is followed by a significant loading on the thymus (Zubkova et al., 2002). According to literature data, the effect of infrared laser radiation on the thymus projection area increase its activity and it is expressed by the stimulation of secretion of $\alpha 1$-thymosin in the thymus epithelial cells (Novoselova et al., 2006). It seems promising to use the additional effect of infrared laser radiation on the projection area of the thymus to maintain immunological and morphogenetic control by the thymus at the final stage of the regeneration process in the traumatized skeletal muscle.

\section{CONCLUSION}

The received data showed that low level radiation of $\mathrm{He}-\mathrm{Ne}$ and IR lasers stimulate regeneration of skeletal muscles, wound healing and post radiation recovery in cells of the thymus and bone marrow after local and total ionizing radiation. Herewith, the reactive changes in the thymus appearanced early and displaied up to the end of observation (60 days), that can point to the increase of function and loading on organ. We suggest that the findings should be considered in clinical practice.

\section{REFERENCES}

Agaiby, A.D., L.R. Ghali, R.Wilson and M. Dyson, 2000. Laser Modulation of angiogenic factor production by T-lymphocytes. Laser Surg. Med., 26(4): 357-363. DOI: 10.1002/(SICI)10969101(2000)26:4<357::AID-LSM3>3.0.CO;2-O

Barilyak, I.R. and I.V. Lopushan, 1981. Cytogenetic consequences of helium-neon laser irradiation of rat bone marrow cells. Patol. Fiziol. Eksperim. Ter., 3: 50-53. PMID: 7279448

Borue, X., S. Lee, J. Grove, E.L. Herzog and R. Harris et al., 2004. Bone marrow-derived cells contribute to epithelial engraftment during wound healing. Am. J. Pathol, 165: 1767-1772. PMID: 15509544

Bulyakova, N.V., S.M. Zubkova, V.S. Azarova, L.V. Mikhaylik and N.I. Varakina, 2002. Effect of Pulsed Laser Radiation on Regeneration of Injured Muscles with Different Regeneration Capacities and the State of the Thymus. Doklady Biol. Sci., 382: 65-70. DOI: 10.1023/A:1014494027033

Doin-Silva, R., V. Baranauskas, L. Rodrigues-Simioni and M.A. de Cruz-Höfling, 2009. The ability of low level laser therapy to prevent muscle tissue damage induced by snake venom. Photochem Photobiol., 85: 63-69. DOI: 10.1111/j.17511097.2008.00397.x

Fedoseeva, G.E., N.K. Smolyaninova, T.I. Karu and A.V. Zelenin, 1988. Human lymphocyte chromatin changes following irradiation with a He-Ne laser. Lasers Life Sci., 2: 197-205. 
Ferrari, G., A.G. Cusella, M. Coletta, E. Paolucci and A. Stornaiuolo et al., 1998. Muscle regeneration by bone marrow-derived myogenic progenitors, Science, 279: 1528-1530. DOI: $10.1126 /$ science. 279.5356 .1528

Golovin, G.V., I.G. Dutkevich and A.P. Sarkisyan, 1978. Effect of laser radiation on the morphological makeup of the peripheral blood and bone marrow experimentally and clinically (a review of the Soviet and foreign literature). Vestn Khir Im I I Grek., 121: 121-120.

Konchugova, T.V., S.B. Pershin, A.A. Minenkov, A.I. Galenchik and A.S. Bobkova, 1992. Immune suppression in local exposures to low-energy infrared laser radiation. Vopr Kurortol Fizioter Lech Fiz Kult. 57-59. PMID: 1413613

McFarlin, K., X. Gao, Y.B. Liu, D.S. Dulchavsky and D. Kwon et al., 2006. Bone marrow-derived mesenchymal stromal cells accelerate wound healing in the rat. Wound Repair and Regeneration, 14: $\quad 471-478$.

DOI: $10.1111 /$ j.17436109.2006.00153.x

Merly, F., L. Lescaudron, T. Rouaud, F. Crossin and M.F. Gardahaut, 1999. Macrophages enhance muscle satellite cell proliferation and delay their differentiation. Muscle Nerve, 22: 724-732. DOI: $10.1002 /($ SICI) $1097-$ 4598(199906)22:6<724::AID-MUS9>3.0.CO;2-O

Mester, E., S. Nagyluckay, S. Tisza and A. Mester, 1978. Stimulation of wound healing by means of laser rays: Investigation of the effect on immune competent cells. Acta. Chir. Acad. Sci. Hung., 121: 121-126. PMID: 735645

Michurina, T.V., G.V. Satdykova, D.K. Voskresenskii, L.N. Gauze, A.I. Chekunova and N.G. Khrushchov, 1991. The bone marrow origin of cells incapsulating a foreign body in peritoneal cavity of xenogeneic radiation chimeras. Ontogenez, 22: 76-83. PMID: 1857589
Novoselova, E.G., O.V. Glushkova, D.A. Cherenkov, V.M. Chudnovsky and E.E. Fesenko, 2006. Effects of low-power laser radiation on mice immunity. Photodermatol. Photoimmunol. Photomed., 22: 3338.

Pagel, C.N., J.E. Morgan, J.G. Gross and T.A. Partridge, 2000. Thymic myoid cells as a source of cells for myoblast transfer. Cell Transplant, 9: 531538. PMID: 11038069

Rappolee, D.A. and Z. Werb, 1992. Macrophage derived growth factors. Current Top Microbiol. Immunol.,181: 87-140. PMID: 1424786

Shefer, G., T.A., Partridge, L. Heslop, J.G. Gross, U. Oron and O. Halevy, 2002. Low-energy laser irradiation promotes the survival and cell cycle entry of skeletal muscle satellite cells. J. Cell Sci., 115:1461-1469. PMID: 11896194

Shefer, G., U. Oron, A. Irintchev, A. Wernig and O. Halevy, 2001. Skeletal muscle cell activation by low energy laser irradiation: a role for MARK/ERK pathway. J. Cell Physiol., 187: 73-80.

Stepanov, B.I., Mostovnikov, V.A., A.N. Rubinov and I.V. Khohlov, 1977. Regulation of functional activity of human cells by laser radiation. Dokl. Akad. Nauk SSSR, 236: 1007-1010. PMID: 579171

Wu, Y., L. Chen, P.G. Scott and E.E. Tredget, 2007. Mesenchymal stem cells enhance wound healing through differentiation andngiogenesis. Stem cells, 25: 2648-2659.

Yarilin, A.A., 1997. Radiation and immunity. A modern look at old problems. Radiation Biology. Radioecology, 37: 597-603. PMID: 9599618

Zubkova, S.M., N.V. Bulyakova, L.V. Mikhaylik, N.I. Varakina and V.S. Azarova, 2002. Histogenetic, metabolic and immunological aspects of the infrared laser radiation on injured irradiated and unirradiated rat skeletal muscle. Radiation Biology. Radioecology, 42: 315-321. PMID: 12125272 